\title{
Atmospheric Effects on Free-Space THz
}

Yihong Yang*

School of Electrical and Computer Engineering, Oklahoma State University, USA

*Corresponding author: Yihong Yang, School of Electrical and Computer Engineering, Oklahoma State University, Stillwater, OK 74078, USA, Tel: 405-744-3085; Email: kaenyyh@gmail.com

Received date: July 22, 2014, Accepted date: July 23 2014, Published date: July 29, 2014

Copyright: (c) 2014 Yang Y. This is an open-access article distributed under the terms of the Creative Commons Attribution License, which permits unrestricted use, distribution, and reproduction in any medium, provided the original author and source are credited.

\section{Editorial}

Terahertz $(\mathrm{THz})$ frequency radiation covers the range from $100 \mathrm{GHz}$ to $10 \mathrm{THz}$ which locates between microwaves and infrared regions. $\mathrm{THz}$ technology has attracted a lot of attentions, and the corresponding applications based on $\mathrm{THz}$ pulses are growing enormously, since interests on nondestructive evaluation, remote detection, wireless communication and security applications, such as sensing and imaging, have increased rapidly. For example, the wireless communication with carrier frequency within the $\mathrm{THz}$ region are very attractive, not only because $\mathrm{THz}$ frequency has the larger frequency bandwidth and higher transmission rate than the current microwave bands, but also has the less scattering loss than optics in the diffusive atmosphere, such as dust and fog. Moreover, the low loss and large bandwidth digital $\mathrm{THz}$ wireless ground link and satellite link are a promising alternative solution to solve the important problem of providing isolated cities, communities, remote industrial, technical and scientific sites with wideband access to the fiber backbone, instead of using the existing microwave ground or satellite links.

One of common properties among above free-space $\mathrm{THz}$ applications is the requirement of $\mathrm{THz}$ pulses propagated through atmosphere. However, $\mathrm{THz}$ pulses suffer attenuation and Group Velocity Dispersion (GVD) from the atmospheric water vapor and scattering loss by suspended particles, such as fog and smoke, especially within the frequency range from 0.05 to $2 \mathrm{THz}$. Consequently, a good understanding of the atmospheric effects including both accurate experimental results and comprehensive theory over broadband $\mathrm{THz}$ range is crucial for most of the free-space $\mathrm{THz}$ applications, which require long distance propagation of $\mathrm{THz}$ pulses through atmosphere, such as ground- and satellite-based radio astronomy, communication links, and remote spectroscopic applications.

\section{THz Time-Domain Spectroscopy (THz-TDS)}

The breakthrough technology to significantly improve $\mathrm{THz}$ research was the invention of THz Time-Domain Spectroscopy (THz-TDS) by Prof. Grischkowsky. The coherent detection associated with femtosecond laser can measure the electrical field of $\mathrm{THz}$ pulses as a function of time by varying the time delay between the laser pulses gated window and $\mathrm{THz}$ pulses at receiver. The coherent detection determines the high signal-to-noise ratio without background noise interruption. The Fourier Transform of measured electrical field provides the complete complex amplitude spectral response of the sample, including absorption and phase shift.

Technology advances had promoted the research of atmospheric water vapor absorption in some aspects. For example, the Fourier Transform Spectroscopy (FTS), Fabry Perrot (FP) resonator have made progress in the study of water vapor effect. However, because of their relatively low sensitivity within $\mathrm{THz}$ frequencies, the technique of THz-TDS becomes more important in this study.

\section{Water Vapor Resonant Absorption}

Firstly, water vapor is one of the most important components in the atmosphere, which is responsible for the atmospheric humidity. Even though water vapor is only one percent of the total atmosphere, at $50 \%$ Relative Humidity (RH) water vapor can account for more than $20 \%$ of the refractivity, which influences the transit time of the propagated $\mathrm{THz}$ pulses. The frequency-dependent absorption $\alpha(\omega)$ and the corresponding dispersive index of refraction $n(\omega)$ of water vapor control the electromagnetic propagation of broad-bandwidth $\mathrm{THz}$ pulses in the atmosphere, because water molecules have several thousand rotational resonant absorption lines from the radio wave range through the terahertz region. (Oxygen gas has a cluster of approximately 25 overlapping lines at $60 \mathrm{GHz}$ and a single line at 120 GHz.) Not only does water vapor absorption attenuates the power of propagated $\mathrm{THz}$ pulse, but the water vapor dispersion reshapes and distorts the propagated $\mathrm{THz}$ pulse severely.

Many simulation codes have been developed to model the absorption of $\mathrm{THz}$ radiation by water vapor in the atmosphere. The total water vapor resonant line absorption can be calculated as the sum of all of the individual resonant lines with corresponding molecular parameters. To date, several professional spectroscopic databases including Molecular Spectroscopy Database in Jet Propulsion Laboratory (JPL), High-Resolution Transmission Molecular Absorption Database (HITRAN) have been created to provide the most accurate values of the parameters, such as intensity, line center frequency and line width, for water vapor molecules and other species. Moreover, HITRAN is the only database to provide the factors of airbroadened width and self-broadened width, which can be used to calculate the line widths of the individual absorption lines, which are also sensitive to the relative pressure and the ambient air pressure. The spectroscopic databases of HITRAN provide 8678 water vapor absorption lines up to $30 \mathrm{THz}$, and 4679 oxygen absorption lines up to $8 \mathrm{THz}$, respectively. The necessity to include strong absorption lines above $2 \mathrm{THz}$ is due to the strong effects on the attenuation and phase from their far wing absorption.

By applying the molecular parameters of water vapor molecule from spectroscopic databases to a proper line shape function, the absorption and phase shift curves of water vapor over broadband $\mathrm{THz}$ frequencies can be calculated. So far, the Molecule Response Theory (MRT) has been experimentally proven to be the most accurate line shape model to well fit the measured absorption line. MRT line shape model includes the molecular orientation time $\tau c$ during a collision and changes smoothly from van Vleck-Weisskopf ( $\mathrm{vV}-\mathrm{W})$ line shape near the line center to Full Lorentz (FL) line shape at far wings of the 
resonant line. The MRT line shape overcomes the disadvantage of the unphysical far-wing of the $\mathrm{vV}-\mathrm{W}$ line shape, as well as the underestimated absorption from the $\mathrm{vV}-\mathrm{W}$ line shape with a cutoff.

From the result of simulation, the frequency spectra below $1 \mathrm{THz}$ are divided into seven regions called transparent $\mathrm{THz}$ windows by water vapor resonant absorption lines, and the attenuation increase as the frequency increase. For atmosphere at $20^{\circ} \mathrm{C}$ and $\mathrm{RH} 58 \%$ which is equivalent to a water vapor density of $10 \mathrm{~g} / \mathrm{m}^{3}$, the power attenuation at $0.3 \mathrm{THz}$ is less than $5 \mathrm{~dB} / \mathrm{km}$, but the power attenuation at $0.85 \mathrm{THz}$ is about $60 \mathrm{~dB} / \mathrm{km}$.

\section{Water Vapor Continuum Absorption}

In additional to the resonant absorption, atmospheric water vapor also introduces another kind of attenuation on $\mathrm{THz}$ spectra is called continuum absorption. The continuum absorption covers the entire broadband frequencies and can be observed within the transparent $\mathrm{THz}$ windows. The continuum absorption cannot be explained by farwing absorption of the resonant lines. It is still not clear that how the continuum absorption is derived. But it has been experimentally verified to have quadratic frequency dependence and to be a combination of two ambient air pressure terms. One term is proportional to the square of the water vapor partial pressure; the other term is proportional to the product of the water vapor partial pressure and the foreign gas partial pressures in the atmosphere.

The empirically determined continuum absorption is usually defined as the difference between the measured total absorption and the calculated absorption of the resonant lines. The determined continuum absorption depends strongly on the line shape function, number of lines, line intensities and line width chosen for the line-byline summation method. In the latest report, the continuum absorption reaches nearly $30 \mathrm{~dB} / \mathrm{km}$ of power attenuation at $0.85 \mathrm{THz}$ at $20^{\circ} \mathrm{C}$ and $\mathrm{RH} 58 \%$ which is half of the corresponding resonant absorption. However, below $0.3 \mathrm{THz}$, the continuum absorption has higher attenuation than resonant absorption.

\section{Suspended Particle and Scintillations}

Besides atmospheric water vapor, different weather conditions bring various particles into atmosphere, such as fog, dust, rain and snow, which also influence the propagated $\mathrm{THz}$ pulses. The physical understanding of this phenomenon is relatively simple, and mostly depends on the comparison between the wavelength and the distributed particle sizes.

Recent work demonstrates that the artificial fog with a visibility of 5 $\mathrm{m}$ attenuates the amplitude of $\mathrm{THz}$ pulses transmitted through $137 \mathrm{~m}$ by only $10 \%$. Based on the observations of fog and cloud droplet size distributions, we can understand the broadband $\mathrm{THz}$ pulses can propagate through dense of atmospheres of fog and clouds, with certain propagation loss, due to Rayleigh scattering. The fog particles are generally considered to be less than 50 microns in diameter, which is much smaller than the $\mathrm{THz}$ wavelengths (300 microns for $1 \mathrm{THz}$ ). This property gives $\mathrm{THz}$ frequency techniques the opportunity for applications such as imaging through dense fog, clouds and fire smoke, and high data rate wireless communication without interruption by fog, cloud and smoke.

For the direct long-path links, it might be expected that scintillations, time varying fluctuations in the refractivity, due to atmospheric temperature and humidity variations, would lead to phase distortions of the transmitted coherent $\mathrm{THz}$ pulses and consequent changes in the incoming beam direction. The effect of turbulence on propagating electromagnetic radiation has been predicted theoretically, and verified experimentally for propagating IR and optical radiation. An experiment measuring intensity and Angle of Arrival (AOA) effects has been done with a $140 \mathrm{GHz}$ beam over path lengths of $1.3 \mathrm{~km}$. Also, for a longer $25 \mathrm{~km}$ path with a $173 \mathrm{GHz}$ beam, using an inter ferrometric radar system capable of measuring deviations of micro-radians, an AOA distribution with standard deviation of only 10 micro-radians was observed. So for a long-path $\mathrm{THz}$ links with carrier frequency below $1 \mathrm{THz}$, scintillation do not appear to be a problem, even though for higher frequencies the problem becomes more severe.

Consequently, $\mathrm{THz}$ radiation absorption from water vapor resonant absorption, continuum absorption and scattering by suspended particles determines the maximum propagation distance of $\mathrm{THz}$ pulses in the atmosphere. The reshaping of propagating broad-bandwidth short $\mathrm{THz}$ pulses is controlled by both the refractivity and the absorption of water vapor resonant dispersion. With this quantitative knowledge of all above introduced atmospheric effects on $\mathrm{THz}$ pulses propagation, we can design free-space $\mathrm{THz}$ applications and analyze their performance based on the atmospheric limits. 